\title{
Transculturalidade na enfermagem baseada na teoria de Madeleine Leininger
}

\author{
Transculturality in nursing based on Madeleine Leininger's theory
}

Transculturalidad en enfermería a partir de la teoría de Madeleine Leininger

Elielson Rodrigues da Silva ${ }^{1 *}$, Emanoela Barros de Alencar ${ }^{1}$, Edvalda Alves Dias ${ }^{2}$, Luciano Crispim da Rocha1, Sabrine Canonici Macário de Carvalho

\section{RESUMO}

Objetivo: Descrever através de uma revisão narrativa a importância do cuidado transcultural baseado na teoria de Madeleine Leininger com inserção na prática do enfermeiro. Revisão Bibliográfica: A Teoria da Diversidade e Universalidade do Cuidado Cultural é a única teoria que tem como enfoque principal o elo entre a cultura, o bem-estar e os cuidados em saúde e no momento atual existem poucos artigos com essa aplicabilidade no Brasil. No entanto, a enfermagem vem empregando esta teoria em vários campos de atuação e vem sendo utilizada em distintas temáticas, o que gera maior relevância para sustentação de sua validação sistemática e continua no país, visto que promove maior conforto ao paciente, preservando sua cultura, religião, etnia e crenças. Considerações Finais: O cuidado transcultural e o processo saúde-doença em enfermagem estão diretamente relacionados com a prática do enfermeiro no ambiente de trabalho, tendo como princípio o respeito pela diversidade cultural de cada indivíduo. Com base nas informações obtidas, a descrição da importância do cuidado transcultural de Leininger, ressalta que, o enfermeiro que insere essa teoria na sua área de trabalho terá mais autonomia na prática do cuidado, trazendo mais conforto ao paciente, preservando a cultura, religião, etnia e crenças do mesmo.

Palavras-chave: Enfermagem transcultural, Teoria de enfermagem, Cultura.

\begin{abstract}
Objective: To describe through a narrative review the importance of cross-cultural care based on Madeleine Leininger's theory with insertion into the nurse's practice. Bibliographic Review: The Culture Care Diversity and Universality is the only theory that has as main focus the link between culture, well-being and health care and at the current moment there are few articles with this applicability in Brazil. However, nursing has been employing this theory in several fields of action and has been used in different themes, which generates more relevance to sustain its systematic and continuous validation in the country, since it promotes greater comfort to the patient, preserving his culture, religion, ethnicity and beliefs. Final Considerations: The transcultural care and the health-disease process in nursing are directly related to the nurse's practice in the work environment, having as principle the respect for the cultural diversity of each individual. Based on the obtained information, the description of the Leininger's transcultural care importance, emphasizes that, the nurse who inserts this theory in his work environment will have more autonomy in the care practice, bringing more comfort to the patient, preserving the patient's culture, religion, ethnicity and beliefs.
\end{abstract}

Keywords: Transcultural nursing, Nursing theory, Culture.

\footnotetext{
${ }^{1}$ Centro Universitário do Rio São Francisco (UniRios), Paulo Afonso - BA.

*E-mail: elielsonfasvipa@gmail.com

2 Faculdade São Vicente de Pão de Açúcar (FASVIPA), Pão de Açúcar - AL.
} 


\section{RESUMEN}

Objetivo: Describir a través de una revisión narrativa la importancia del cuidado transcultural basado en la teoría de Madeleine Leininger con inserción en la práctica de enfermeras. Revisión Bibliográfica: La Teoría de la Diversidad y Universalidad del Cuidado Cultural es la única teoría que tiene como foco principal el vínculo entre cultura, bienestar y cuidado de la salud y en este momento hay pocos artículos con esta aplicabilidad en Brasil. Sin embargo, la enfermería ha venido utilizando esta teoría en varios campos de acción y ha sido utilizada en diferentes temáticas, lo que genera mayor relevancia para sustentar su validación sistemática y continúa en el país, ya que promueve un mayor confort al paciente, preservando su cultura, religión, etnia y creencias. Consideraciones Finales: El cuidado transcultural y el proceso salud-enfermedad en enfermería están directamente relacionados con la práctica del enfermero en el ámbito laboral, con el principio de respeto a la diversidad cultural de cada individuo. Con base en la información obtenida, la descripción de Leininger sobre la importancia del cuidado transcultural enfatiza que el enfermero que inserta esta teoría en su área de trabajo tendrá más autonomía en la práctica del cuidado, brindando mayor comodidad al paciente, preservando la cultura, la religión etnia y creencias.

Palabras clave: Enfermería transcultural, Teoría de enfermería, Cultura.

\section{INTRODUÇÃO}

A jornada da enfermagem, no decorrer de seus acontecimentos, passou de uma missão aprendida para uma profissão, do método de preparo e atuação cêntrica voltada para o âmbito hospitalar para a universidade, da simples obediência para a responsabilidade e autonomia, e de uma aplicação executada a uma fundamentação teórica (PETERSEN CB, et al., 2016).

No que diz respeito ao campo das profissões voltadas para a assistência à saúde, a enfermagem sustentase atualmente como uma profissão que zela com o cuidado cedido ao ser humano, em diversos ambientes e contextos de cuidado em saúde que são inerentes as culturas distintas. Em virtude disso, é de responsabilidade da enfermagem estudar as teorias para favorecer uma prática mais eficiente e resolutiva. Salientando que, estudar as teorias não significa dizer que é necessário aceitá-las integralmente, mas é possível usá-las como uma base de conhecimento próprio, adequando os conteúdos teóricos a prática (HENCKEMAIER L, et al., 2014).

Nos últimos decênios, autores de enfermagem de diversos países têm se impressionados em relação ao cuidado e os fatores culturais. Na década de 50, Madeleine Leininger ao trabalhar em uma casa com crianças de diversas nacionalidades, observou que as mesmas precisavam do cuidado de forma diferenciado, surgindo assim a Teoria Transcultural do Cuidado (TCC). Essa teoria conceitua o cuidado universal ao ser humano, que ao se desenvolver e morrer, necessita de cuidados adequados ao seu ambiente, cultura e estrutura social, tendo sua própria dimensão de cuidado, doença e saúde (BOEHS AE, 2002).

É importante ressaltar que em 1978, Madeleine Leininger mostrou sua teoria em um livro-base datado no referente ano, continuando após muitos capítulos de livros e artigos. No ano de 1991 uma nova versão de seu livro-base foi publicada por ela, com alguns ajustes, principalmente modificações em conceitos prévios e relacionados à operacionalização da teoria. Somente em 1994 existiram maiores números de publicações em dissertações que refletiram em bibliografias atualizadas sobre essas teorias, inclusive houve o aumento de publicações brasileiras (BOEHS AE, et al., 2010).

A Teoria de Leininger no Brasil tem aumentado à aliança das enfermeiras na antropologia. Certamente usar uma teoria com princípios da antropologia e da enfermagem, ajuda na união dos mundos: cliente e profissional de saúde, e impulsiona o saber na enfermagem. Para que isso se realize, é necessário ser cuidadoso, para não realizar uma aplicação da teoria de maneira repetitiva, sem observações críticas, pois assim como outras abordagens com base na antropologia, a Teoria de Leininger possui limitações e ambiguidades de termos que precisam ser esclarecidos (BOEHS AE, 2002). 
Entretanto, a teoria contribui para uma proposta do cuidado como um todo, que conceitua a preservação dos sujeitos e a variedade de fatores culturais, em contestação ao modelo hegemônico centrado nos sinais e sintomas das doenças. A educação em saúde facilita que a população use seus próprios saberes conquistados para buscar resoluções para seus problemas (SEIMA MD, et al., 2011).

A aplicação de uma teoria para conduzir a assistência de enfermagem permite que esta seja desigualada e com fundamento científico, realizando um cuidado diferente do cuidado habitual. Inserir no planejamento da assistência de enfermagem os saberes antecedentes de indivíduos a serem cuidados e seus familiares consente maior elo das equipes de enfermagem à comunidade, com visão em uma assistência mais concreta e reflexiva com as atitudes, crenças e valores dos seres humanos que procuram os cuidados para suprir suas necessidades dentro do processo saúde-doença (HENCKEMAIER L, et al., 2014).

Nessa perspectiva, a escolha deste tema surgiu da observação durante experiência prática no campo de estágio com as dificuldades dos enfermeiros de lidar com culturas diferentes, corroborado na literatura, no intuito de levantar evidências científicas sobre os cuidados transculturais relacionados ao processo saúdedoença e associar os cuidados de enfermagem que possam conduzir melhor a terapêutica do cliente e o respeito individual de sua cultura, proporcionando assim uma assistência holística.

O presente estudo descreve as evidências científicas em torno das práticas do cuidado cultural visando estreitar as lacunas do conhecimento e contribuir para a melhoria da qualidade da assistência de enfermagem. Estes fatores são de relevância social, científica e profissional. Assim, percebe-se a relevância do presente estudo, pois pretende-se implementar a Teoria da Diversidade e Universalidade do Cuidado Cultural (TDUCC) na prática assistencial do trabalho do enfermeiro direcionado ao cuidado dos clientes com diversas culturas distintas. Dessa forma o estudo objetiva descrever através de uma revisão narrativa a importância do cuidado transcultural baseado na teoria de Madeleine Leininger com inserção na prática do enfermeiro.

\section{REVISÃO BIBLIOGRÁFICA}

Com base na análise de conteúdo temático categorial, foi possível destacar quatro categorias: Teoria da diversidade e universalidade do cuidado cultural de Madeleine Leininger; $A$ transculturalidade no processo saúde-doença; O conhecimento transcultural e a atuação da enfermagem; A implantação da sistematização da assistência de enfermagem no cuidado cultural.

\section{Teoria da diversidade e universalidade do cuidado cultural de Madeleine Leininger}

A TDUCC foi criada por Leininger, precursora da enfermagem transcultural. A partir dos anos 50, a mesma vem estudando como empregar este conhecimento na atuação da enfermagem. Baseando-se nas disciplinas de Sociologia, Psicologia e Antropologia elaboraram a TDUCC, tendo como principal proposta, promover cuidados, num comportamento holístico de acordo com a cultura, com ênfase na enfermagem cientifica e humanística. O cuidado cultural procura compreender a diversidade e universalidade do cuidado humano em relação à visão de mundo, estrutura social e outras dimensões e assim, descobrir o modo de prover cuidados culturalmente congruentes para diferentes pessoas, famílias ou grupos culturais (QUEIROZ MVO e PAGLIUCA LMF, 2001).

A Norte-americana enfermeira, Madeleine Leininger, publicou a teoria da Diversidade e Universalidade do Cuidado Cultural no ano de 1985, sua teoria foi levantada a partir do ponto de vista, que os povos de cada cultura são instruídos a conhecer e interpretar as formas, cujo eles testam e compreendem seu cuidado (RAMOS JLC e MENEZES MR, 2012). Essa teoria influenciou a categoria da enfermagem brasileira com a finalidade de aliar os aspectos culturais dos seus pacientes e ofertar um cuidado adequado à cultura deles (BETIOLLI SE, et al., 2013).

Leininger desenvolveu uma metodologia na qual sua teoria pode ser usada na prática das medidas de cuidados. Dessa forma indica que as realizações de cuidados têm o objetivo de ajudar e conduzir as ações e resoluções de enfermagem através da preservação ou manutenção do cuidado cultural e reforma ou repadronização do cuidado cultural (HENCKEMAIER L, et al., 2014). 
No Brasil, a teoria Transcultural tem aproximado mais os enfermeiros da antropologia. Certamente, aplicar uma teoria com embasamento na antropologia e na enfermagem é um favorecimento para interligar o mundo do profissional com o mundo do cliente e incrementar o saber na enfermagem (MOURA MAV, et al., 2005).

O eixo principal da Teoria Transcultural idealiza o cuidado como o fundamento da prática e do saber. Por isso, pretende assistir, oferecer auxílio e facilitar ações para suprir as necessidades. Em vista disso, para Leininger, o cuidado que é primordial a vida é também um ato cultural. Desse modo, entende-se que cada povo tem seu jeito próprio de se cuidar (LOPES WMPS e FIGUEIREDO MLF, 2011).

Cuidados de enfermagem aceitáveis habitualmente, de acordo com a TDUCC, favorecem a saúde da pessoa, família, grupos e suas comunidades, nos diversos ambientes. A população que faz uso dos serviços de saúde sente-se mais repleta com os profissionais que valorizam e respeitam o seu modo de viver. A veracidade da enfermagem brasileira é extensão para executar a teoria tendo em vista a dessemelhança regional de circunstâncias sócias e culturais que desencadeiam diferentes imposições, significados e expectativas de cuidado (SEIMA MD, et al., 2011).

A teoria se fundamenta na confirmação de que "em todas as culturas o cuidado é primordial para sustentação e de fortalecimento da saúde e a persistência da vida humana". As implementações do cuidado cultural proporcionais com as crenças, valores e costumes da pessoa, auxiliando-o de maneira relevante e ordeiro no tempo existencial até a morte (MARTINS PAF e AIVIM NAT, 2012). O cuidado transcultural referese a uma teoria que busca aproximar os aspectos da vida do ser humano e suas complicações, vendo as pessoas como seres de relações (HENCKEMAIER L, et al., 2014).

\section{A transculturalidade no processo saúde-doença}

A saúde resulta de um seguimento de construção social, que sofre interferência das situações vividas e do acesso a bens e serviços, sendo edificado individualmente ou coletivamente, por ações de governo, da população e de cada indivíduo (SECCO AC, et al., 2017).

Soares NA, et al. (2014) ressalta que estudos recentes direcionam a saúde-doença das pessoas a cultura, no qual a saúde é a soma das relações socioculturais que a sociedade impõe em seu convívio cotidiano. Frente a essa realidade, os programas governamentais de controle devem considerar o conhecimento da população, pois esses podem influenciar nas condutas para o controle de doenças.

O processo saúde-doença é entendido como a procriação do homem sobre o processo de adoecimento. $O$ aspecto como o ser humano entende-se saudável, ou as condições pelas quais o mesmo alcança "o estar saudável", "estar adoecendo" ou ainda "estar doente" está entendida dentro da ótica cultural (SILVA JLL, et al., 2013).

Foi lançando em 2003 a Política Nacional de Humanização (PNH) como reforço da atenção, que tenta inserir em prática os princípios do SUS na rotina dos serviços destinados a saúde propondo modificações nas maneiras de coordenar e cuidar à Secretaria de Atenção à Saúde do Ministério da Saúde é associado ao PNH no Departamento de Ações Programáticas e Estratégicas (DAPES), compondo de maneira distribuída, planos e ações para proporcionar e espalhar aperfeiçoamento em saúde, como a filosofia de Clínica Ampliada, em que o cuidar em saúde não se refere somente de um nível de atenção do sistema de saúde, como a ampliação da filosofia clinica ampliada, se refere a uma ação integral que tem sentidos e significados direcionados para o entendimento de saúde como o direito de ser (ALCANTARA LS, et al., 2014).

Nessa perspectiva, a TCC sugere mostrar relativamente à organização coletiva no mundo e outras proporções, as alternativas de aplicar o cuidado culturalmente a população de culturas distintas (diversidade) ou similar (universalidade), com disposição de preservar ou retornar o bem-estar (saúde), ou encarar a doença de forma culturalmente apropriada (CAMARGO FCM, et al., 2014).

\section{$O$ conhecimento transcultural $e$ a atuação da enfermagem}

A profissão de enfermagem no Brasil atua como maior força de trabalho na área da saúde e contribui de forma decisiva no Sistema único de Saúde (SUS), por intermédio, gestão, educação, investigação são eixos que constituem a atividade profissional (ANDRADE SR, et al., 2016). 
Entendendo o cuidado de enfermagem como um acontecimento propositado, primordial a vida, que acontece na relação humana, associa-se a prática do cuidado com a responsabilidade ética que segue as obrigações profissionais, transcendendo o senso comum de uma dedicação pontual para estabelecer-se em uma atenção continuada, sistêmica e contextual, o que inclui as questões culturais (ANDRADE SR, et al., 2016).

Segundo Souza NMG, et al. (2012), o cuidado cultural é definido como valores, crenças e expressões uniformes, relativamente conhecidas, que ajudam, dão suporte ou preparam outras pessoas para estabelecer o bem-estar, favorecer uma melhor condição de vida humana ou auxiliar no processo de aceitação da morte ou das incapacidades físicas.

A descrição da competência cultural como um processo contínuo do ser humano se empenha para se transformar no decorrer do tempo, ser mais autoconsciente, para dar valor a diversidade e tornar-se um conhecedor sobre os aspectos mais relevantes da cultura. A enfermagem como profissão tem adotado esse conceito.

Os enfermeiros explicam a competência cultural como a possibilidade de entender as diferenças culturais, com a finalidade de realizar cuidados de qualidade a uma disparidade de pessoas. Culturalmente os enfermeiros mais sensíveis às questões inerentes com etnia, raça, cultura, gênero e orientação sexual, são os enfermeiros mais competentes. Além disso, os enfermeiros que possuem competência cultural aperfeiçoam com eficiência a capacidade de comunicação, perspectivas culturais e capacidade de conhecimentos ligados com as práticas de saúde de dessemelhantes culturas (VILELAS JM e JANEIRO SID, 2012).

A Enfermagem Transcultural é definida como um subcampo, ou seja, um ramo que se dedica do estudo comparativo e da análise de culturas que tem relação á enfermagem e as práticas de cuidados de saúdedoença, com a proposta oferecer um serviço de atendimento de Enfermagem, eficaz e significativo, para a população, concordando com seus valores culturais e seu contexto de saúde-doença (CAMARGO FCM, et al., 2014).

Entretanto, mesmo com a existência de uma teoria de enfermagem direcionada para a cultura dos povos, ainda há a necessidade por parte de alguns profissionais de enfermagem de compartilhar conhecimentos para execução de uma prática diferente. $O$ entendimento que as pessoas possuem do cuidado é relativo as suas necessidades e segue o seu estilo de viver. De forma que, constituir conversas e relações de auxílio, enquanto a execução das ações que inclui o cuidado de enfermagem, são percurso para que se consiga descobrir com o outro e compreender as suas perspectivas (BUDÓ MLD, et al., 2016).

Torna-se útil integrar o cuidado a cultura do paciente para que o mesmo possa integrá-lo ao seu dia a dia. $\mathrm{O}$ indivíduo entende de modo mais eficiente o cuidado executado bem como o autocuidado, quando os dois se interagem de forma coerente ao modo de vida, e não se contrariando (SILVA JLL, et al., 2013).

A enfermagem é um acontecimento cultural, que engloba processos que auxiliam os indivíduos de dessemelhantes culturas, no qual as pessoas têm seus valores e crenças considerados, sem serem afastada de seu contexto sociocultural. Assim, confia-se que o envolvimento da enfermeira com o cuidado transcultural pode acontecer de forma horizontal, dividindo experiências, no agir, no ouvir e no pensar (BUDÓ MLD, et al., 2016).

O cuidado é baseado na cultura, pois cada povo tem sua cultura própria e sua forma de compreender e praticar a ação do cuidar, ressaltando-se como a diversidade do cuidado. Nesse contexto, para execução de uma assistência satisfatória, o profissional enfermeiro pode examinar os desempenhos de cuidados culturais, determinando com o paciente os cuidados são convenientes, alteráveis ou negociáveis (SILVA JLL, et al., 2013).

A teoria de enfermagem transcultural é complexa, o enfermeiro para aplicá-la deve ter disposição de relacionar-se com pessoas, verificando os valores culturais do indivíduo a ser cuidado e para que possa ser aplicado ele precisa se autoavaliar, para verificar se está apto para aceitar a relação de cuidador frente ao indivíduo a ser cuidado em dessemelhantes cenários e contextos. Diante da autoavaliação o enfermeiro deve levar em consideração os aspectos como os conflitos éticos e raciais, problemas religiosos e sociais e obstáculos físicos e mentais (PAGLIUCA LMF e MAIA IER, 2012). 
Madeleine Leininger demostrou que os profissionais enfermeiros necessitavam conseguir um entendimento mais aperfeiçoado das distintas culturas com a finalidade de executar cuidados a indivíduos de várias etnias. Sendo que nem todos os enfermeiros possuem conhecimentos acerca da cultura relativa ao estado de saúde dos pacientes. Desse modo, surge a necessidade dos mesmos adquirirem conhecimentos e competência intercultural que os auxiliarão a executar cuidados de forma individual, com embasamento nas práticas culturais (VILELAS JM e JANEIRO SID, 2012).

Por conseguinte, a TDUCC é a única teoria que tem como enfoque principal o elo entre a cultura, o bemestar e os cuidados em saúde e no momento atual existem poucos artigos com essa aplicabilidade no Brasil. No entanto, a enfermagem vem empregando esta teoria em vários campos de atuação e vem sendo utilizada em distintas temáticas, o que gera maior relevância para sustentação de sua validação sistemática e continua no país (CAMARGO FCM, et al., 2014).

\section{A implantação da sistematização da assistência de enfermagem no cuidado cultural}

A enfermagem como ciência, até então busca a sustentação dos seus valores profissionais, com o propósito de que o profissional enfermeiro possa realmente criar sua identidade na área da assistência e revelar convicções e posturas, rejeitando o uso de intervenções ao acaso, sem justificativa cientifica, planejamento e reflexão (MARIA MA, et al., 2012).

No Brasil, a consulta de enfermagem teve início na metade dos anos 60 e sua legalização aconteceu por volta da década de 80, mediante da lei oㅜ 7.498/86, que regeu o Exercício da Enfermagem e padronizou essa atividade sendo privativa do enfermeiro. Durante a década de 90, o Conselho Federal de Enfermagem (COFEN) instituiu a obrigação na execução da consulta de enfermagem em todos os níveis de assistência à saúde, no âmbito das intuições privadas ou públicas, de acordo com a Resolução no 159/COFEN (ROCHA GST, et al., 2015).

Tendo início na década de 1950, a SAE tem possibilitado a equipe de enfermagem planejar e direcionar suas ações as necessidades individuais de cada paciente, de forma holística. Torna-se imprescindível nas instituições de saúde, onde são realizadas ações de enfermagem, com objetivo de ofertar o cuidado humanizado a população em estado de doença. Fundamentado por resoluções do COFEN, o Processo de Enfermagem (PE), por intermédio da $\mathrm{SAE}$, permite que assistência seja planejada para atingir as necessidades individuais do cliente, com ênfase no processo holístico, no qual as intervenções sejam elaboradas de forma individual (SILVA PO e PORTELLA VC, 2014).

Dessa forma, compreende-se que a Sistematização da Assistência de Enfermagem (SAE) é um trajeto de autonomia para a enfermagem por corresponder a uma metodologia de assistência assumida pelos enfermeiros, possibilitando uma relação do enfermeiro junto ao cliente, tanto no tempo de sua preparação quanto na execução do cuidado, demandando conhecimento científico e responsabilidade profissional (MENEZES SRT, et al., 2011).

A implantação da SAE, como prática de um processo de tarefa conveniente as carências da comunidade e como modelo de assistência a ser executado pelo enfermeiro em todas as áreas que abrange a assistência à saúde, promove, com mais precisão, melhor qualidade da assistência de enfermagem. Diante disso o COFEN constituiu a Resolução no 272/026, modificada pela Resolução no 358/097, que aplica a sistematização de enfermagem nas instituições de saúde do Brasil (MEDEIROS SRT, et al., 2013).

A SAE tem sido executada em distintas entidades de saúde, e forma uma metodologia cientifica no qual o enfermeiro estabelece para utilizar seus saberes técnico científico humanizados na prestação de assistência aos clientes. Facilita a organização do trabalho em saúde por meio da realização do processo de enfermagem, o qual pode ser compreendido como a utilização de uma teoria de enfermagem na assistência aos clientes (GANDOLFI M, et al., 2016).

Dessa forma, por meio da SAE é possível elaborar diagnósticos de enfermagem, entre eles, os diagnósticos relacionados à cultura, sendo utilizados durante a consulta de enfermagem, anamnese e registro de evolução de paciente, promovendo assim uma assistência de enfermagem mais holística, com maior oferta do cuidado e com melhores resultados. 
Isto posto, os diagnósticos de enfermagem propostos pela NANDA possuem como objetivo fornecer embasamento científico à prática do cuidado, buscando intervenções de enfermagem direcionadas ao controle de resultados positivos que norteiam a prática, promovendo bem-estar e qualidade de vida.

Entre esses diagnósticos, existem alguns diagnósticos de enfermagem relacionados à cultura e pautados no embasamento científico, sendo eles (NANDA INTERNACIONAL, 2018): Campo de energia perturbado: relacionado a desaceleração ou bloqueio dos fluxos de energia, caracterizado por mudança de temperatura (calor/frio); Comunicação verbal prejudicada: relacionado a diferenças culturais, caracterizado por incapacidade de falar o idioma do cuidador; Controle familiar ineficaz do regime terapêutico: relacionado às dificuldades econômicas, caracterizados por dificuldade com o regime terapêutico; Desesperança: relacionado à perda da crença não poder espiritual, caracterizado por alterações no padrão do sono; Religiosidade prejudicada: relacionado a crise espiritual, caracterizado por questionar padrões de crenças religiosas; Fadiga: relacionado a fatores ambientais: barulho, luzes, temperatura, caracterizado por incapacidade de manter as rotinas habituais.

\section{CONSIDERAÇÕES FINAIS}

Com base nas informações obtidas, a descrição da importância do cuidado transcultural da TDUCC de Leininger, ressalta que, o enfermeiro que insere essa teoria na sua área de trabalho terá mais autonomia na prática do cuidado. Nesse contexto, conclui-se que é de fundamental importância a implementação dessa teoria na prática do enfermeiro, visto que considera não só o indivíduo, mas a bagagem cultural que ele carrega, relacionando assim cultura, bem-estar e cuidados em saúde. Além disso, por meio do emprego dessa teoria na prática assistencial é possível promover maior conforto ao paciente, preservando sua cultura, religião, etnia e crenças, influenciando positivamente no processo de cuidado em saúde.

\section{REFERÊNCIAS}

1. ALCANTARA LS, et al. Interdisciplinaridade e Integralidade: a Abordagem do Assistente Social e do Enfermeiro no INCA. Revista Brasileira de Cancerologia, 2014; 60(2): 109-118.

2. ANDRADE SR, et al. Fundamentos normativos para a prática do cuidado realizado pela enfermagem brasileira. Revista Brasileira de Enfermagem, 2016; 69(6): 1082-90.

3. BETIOLLI SE, et al. Decisões e ações de cuidados em enfermagem alicerçadas em madeleine leininger. Cogitare Enfermagem, 2013; 18(4): 775-81.

4. BOEHS AE. Análise dos conceitos de negociação/acomodação da teoria de M. Leininger. Revista Latino-Americana de Enfermagem, 2002; 10(1): 90-6.

5. BOEHS AE, et al. Conceitos da teoria do cuidado cultural em dissertações de mestrados. Revista Rene. Fortaleza, $2010 ; 11(4): 630-637$.

6. BUDÓ MLD, et al. Cuidado e cultura: uma interface na produção do conhecimento de enfermagem. Revista Fundamental Care. Online, 2016; 8(1): 3691-3704.

7. CAMARGO FCM, et al. A aplicabilidade da teoria do cuidado cultural por enfermeiras nos periódicos de saúde do brasil (1992- 2011). Revista de Pesquisa: cuidado é fundamental online, 2014; 6(4): 1743-1755.

8. GANDOLFI M, et al. Sistematização da assistência de enfermagem: da teoria ao cuidado integral. Revista de Enfermagem da Universidade Federal de Pernambuco on line, 2016; 10(supl.40): 3694-703.

9. HENCKEMAIER L, et al. Cuidado Transcultural de Leininger na Perspectiva dos Programas de Pós-Graduação em Enfermagem: Revisão Integrativa. Revista ciência e saúde, 2014; 7(2): 85-91.

10. LOPES WMPS, FIGUEIREDO MLF. O cuidado transcultural como base para investigar idosas mastectomizadas sobre o conhecimento e o uso de sutiãs e próteses externas. Revista Enfermagem em Foco, 2011; 2(supl): $81-84$.

11. MARIA MA, et al. Sistematização da assistência de enfermagem em serviços de urgência e emergência: viabilidade de implantação. Revista Brasileira de Enfermagem, 2012; 65(2): 297-303.

12. MARTINS PAF, AIVIM NAT. Plano de cuidados compartilhados: convergência da proposta educativa problematizada com a teoria do cuidado cultural de enfermagem. Revista Brasileira de enfermagem, 2012; 65(2): 368-73.

13. MEDEIROS AL, et al. Sistematização da assistência de enfermagem: dificuldades evidenciadas pela teoria fundamentada nos dados. Revista de enfermagem da Universidade do Estado de Rio de Janeiro, 2013; 21(1): 47-53.

14. MENEZES SRT, et al. Autonomia e vulnerabilidade do enfermeiro na prática da Sistematização da Assistência de Enfermagem. Revista da Escola de Enfermagem da Universidade de São Paulo, 2011; 45(4): 953-8.

15. MOURA MAV, et al. Teoria transcultural em pesquisas de enfermagem. Revista Escola Anna Nery Revista de Enfermagem, 2005; 9(3): 434-40.

16. NANDA INTERNACIONAL. Diagnósticos de enfermagem da NANDA: definições e classificação 2018-2020. Porto Alegre: Artmed, 2018. 
17. PAGLIUCAI LMF, MAIA IER. Competência para prestar cuidado de enfermagem transcultural à pessoa com deficiência: instrumento de autoavaliação. Revista Brasileira de Enfermagem, 2012; 65(5): 849-55.

18. PETERSEN CB, et al. Necessidades de saúde e o cuidado de enfermagem. Revista Brasileira de Enfermagem, 2016; 69(6): 1236-9.

19. QUEIROZ MVO, PAGLIUCA LMF. Conceito de enfermagem transcultural: análise de seu desenvolvimento em uma dissertação de mestrado. Revista Brasileira de Enfermagem, 2001; 54(4): 630-637.

20. RAMOS JLC, MENEZES MR. Cuidar de idosos com doença de Alzheimer: um enfoque na teoria do cuidado cultural. Revista da Rede de Enfermagem do Nordeste, 2012; 13(4): 805-15.

21. ROCHA GST, et al. Prática educativa do enfermeiro na consulta de enfermagem à criança na perspectiva de Madeleine Leininger. Revista de Enfermagem da Universidade Federal do Piauí, 2015; 4(2): 124-9.

22. SECCO AC, et al. Os Grupos como dispositivo de cuidado na AB para o trabalho com pacientes portadores de Diabetes e Hipertensão. Revista. Mudanças - Psicologia da Saúde, 2017; 25(1): 9-15.

23. SEIMA MD, et al. A produção científica da enfermagem e a utilização da teoria de madeleine leininger: revisão integrativa 1985 - 2011. Escola Anna Nery Revista de Enfermagem, 2011; 15 (4): 851-857.

24. SILVA JLL, et al. Reflexões sobre o cuidado transcultural e o processo saúde doença: contribuições para a assistência em enfermagem. Revista de pesquisa: cuidado é

25. fundamental online, 2013; 5(1): 3185-95.

26. SILVA PO, PORTELLA VC. Intervenções de enfermagem na dor. Revista Dor. São Paulo, 2014; 15(2): 145-8.

27. SOARES AN, et al. Crenças e práticas de saúde no cotidiano de usuários da rede básica de saúde. Revista Enfermagem da Universidade do Estado do Rio de Janeiro, 2014; 22(1): 83-8.

28. SOUZA NMG, et al. Visão do mundo, cuidado cultural e conceito ambiental o cuidado do idoso com diabetes mellitus. Revista Gaúcha de Enfermagem, 2012; 33(1): 139-46.

29. VILELAS JM, JANEIRO SID. Transculturalidade: o enfermeiro com competência cultural. Revista Mineira de Enfermagem, 2012; 16(1): 120-127. 